
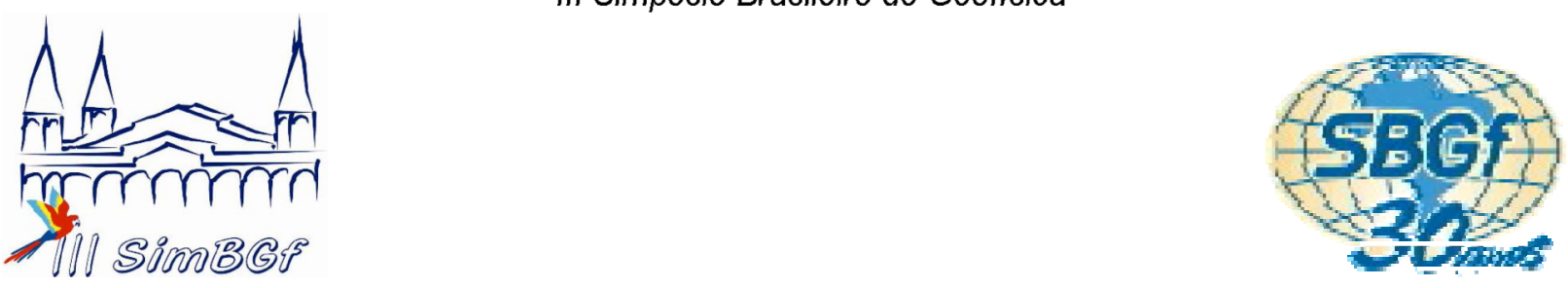

\title{
Variação temporal da resistividade elétrica em contaminação por gasolina
}

Leonardo Zani Castello, César Augusto Moreira, Antônio Celso de Oliveira Braga, Maíra Tozzi Cardinali, Instituto de Geociências e Ciências Exatas, IGCE/UNESP - Campus de Rio Claro.

Copyright 2008, SBGf - Sociedade Brasileira de Geotisica

Este texto foi preparado para a apresentação no III Simpósio Brasileiro de Geofísica, Belém. 26 a 28 de novembro de 2008. Seu conteúdo foi revisado pelo Comitê Técnico Belém, 26 a 28 de novembro de 2008. Seu conteúdo foi revisado pelo Comitê Técnico
do III SimBGf, mas não necessariamente representa a opinião da SBGf ou de seus associados. É proibida a reprodução total ou parcial deste material para propósitos associados. E proibida a reprodução total

\section{Resumo}

Este trabalho visa avaliar a variação temporal do parâmetro físico resistividade elétrica num experimento em escala de laboratório, constituído por tanque com solo areno-argiloso que será alterado por uma contaminação induzida de gasolina. O estudo do comportamento deste derivado de petróleo em escala laboratorial visa produzir subsídios técnicos para melhor compreensão de resultados acerca da caracterização geoelétrica em estudos de casos em áreas contaminadas pelos mesmos tipos contaminantes, e que, não raro apresentam resultados conflitantes.

\section{Introdução}

O crescente consıımn dos derivadns de netrólen e, conseqüentemente, a necessidade de se aumentar à estocagem e a distribuição desses produtos, geraram um aumento relevante nos casos de vazamentos de tanques subterrâneos para armazenamento de combustíveis, o que torna imprescindível o conhecimento das interações entre esses possíveis contaminantes e o meio físico.

Visando detectar e quantificar tais vazamentos, são aplicados atualmente, diferentes técnicas de investigação como: sondagem direta com análise química de hidrocarbonetos totais de petróleo (TPH) em solo e/ou água subterrânea, elaboração de malhas de sondagem para obtenção de dados sobre compostos voláteis (VOCs), além de métodos geofísicos.

A utilização de métodos geofísicos constitui uma imnnrtante ferramenta de annin anc ectuidnc amhientaic, representando um significativo avanço científico. Esses métodos são utilizados principalmente nas fases de prevenção, para caracterização geológica e identificação de áreas vulneráveis - elevada permeabilidade; avaliação preliminar e diagnóstica, caracterização de áreas contaminadas; monitoramento, avaliação temporal dos contaminantes para controle de fluxo, redução dos teores e possíveis alterações físico-químicas resultantes da dispersão, diluição e degradação dos compostos.

A residência de determinados tipos contaminantes em subsuperfície, como compostos inorgânicos como o chorume proveniente de aterros de resíduos sólidos ou compostos orgânicos derivados de petróleo como gasolina e organoclarados, altera significativamente os valores naturais dos principais parâmetros físicos dos materiais geológicos. Estes parâmetros são passíveis de medição pelo instrumental geofísico e, conseqüentes alterações pela presença de compostos contaminantes, permitem a caracterização de anomalias em relação aos parâmetros físicos

\section{Caracterização geoelétrica de hidrocarbonetos no ambiente geológico}

A grande maioria dos autores correlaciona a redução nos valores de resistividade em meios contaminados por hidrocarbonetos à atividade de biodegradação da matéria orgânica por organismos vivos, como bactérias aeróbicas e anaeróbicas (Sauck et al., 2000; Atekwana et al., 2000; Werkema ef al 2nก.3. Atekwana ef al 2nก4. Vena ef al. 2003)

Segundo Atekwana et al., 2000, locais onde ocorre uma significante alteração química do hidrocarboneto, existe uma mudança na assinatura geoelétrica de resistiva para condutiva e afirmam que é possível que a existência dessa zona condutiva possa ser devida, em parte, pela biodegradação do contaminante.

A partir da aplicação do método de eletrorresistividade para analise da atividade de biodegradação em local rontaminadn nor hidrncarhnnetnc nor W/erkema et al. (2003), são destacadas a presença de grandes quantidades de bactérias degradadoras de óleo diesel em áreas com de baixos valores de resistividade elétrica. Os autores sugerem que a magnitude do parâmetro físico representa uma medida indireta da atividade biológica.

A aplicação combinada da técnica de Caminhamento Flétrinn a Snndanem Flótrina Vertiral em I ima of al. (1995), descreve intervalos de baixa resistividade com áreas de concentração de contaminantes provenientes de um aterro de resíduos líquidos derivados de petróleo.

A caracterização geoelétrica de áreas contaminadas por derivados de petróleo sugere anomalias tanto de elevada resistividade como de baixa resistividade. Esta aparente incongruência pode ser esclarecida por meio da correlação do tempo de residência dos contaminantes no solo e tempo de exposição a processos de degradação. Alguns trabalhos sugerem que a biodegradação de compostos (LNAPL) pode mudar as propriedades geoquímicas do aqüífero de forma acentuada, que podem chegar a alterações significativas em medidas de resistividade e na propagação de ondas eletromagnéticas 
(Sauck, 2000; Atekwana et al., 2000). Estudos em escala de laboratório sugerem que a biodegradação de LNAPL sob condições aeróbicas e anaeróbicas podem mudar de forma substancial as condições biogeoquímicas do aqüífero impactado, resultando em reduções drásticas de resistividade elétrica (Cassidy, et al., 2001).

Atenuação Natural compreende um trabalho de remediação que inclui uma variedade de processos físicos, químicos e biológicos que, dentro de condições favoráveis, permite a redução de massa, toxicidade, mobilidade, volume ou concentrações de contaminantes no solo ou água subterrânea sem intervenção humana (EPA, 2004). Este processo é importante para a degradação de derivados de petróleo, por organismos encontrados em quase todos os ambientes.

Vários compostos de fase líquida leve não aquosa (LNAPL), entre eles os benzeno, tolueno, etilbenzeno e xileno, que constituem a gasolina podem ser biodegradados sob condições ambientais (EPA, 1998). Contudo, este processo natural pode ser limitado pela falta de nutrientes, elétrons receptores ou ambos (Thomas \& Ward, 1989). Em muitos locais, o processo de biodegradação fica limitado a zonas periféricas da pluma contaminante, pois a presença de contaminante em elevadas concentrações torna 0 ambiente hostil a proliferação de microorganismos (Wilson \& Jones, 1993; Rabus \& Heider, 1998) (Figura 1)

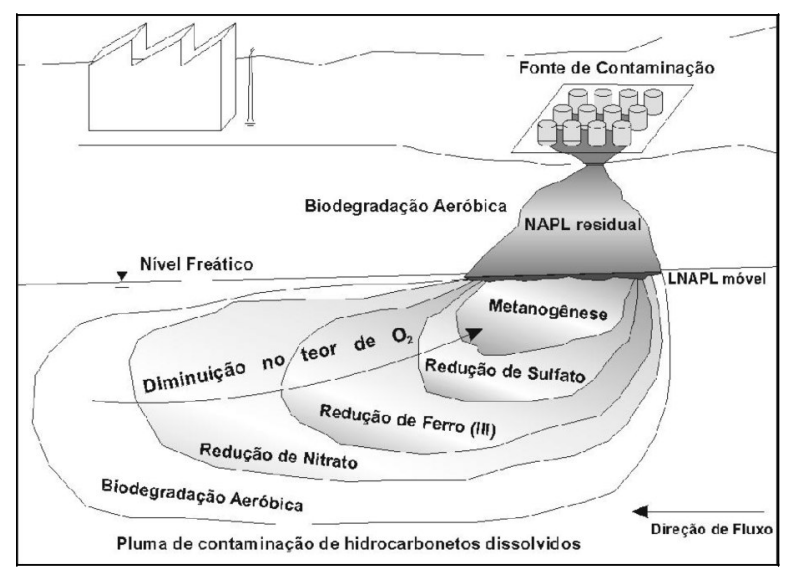

Figura 1 - Zonas de degradação de hidrocarbonetos (adaptado de Rabus \& Heider, 1998)

\section{Metodologia de Pesquisa}

Com o objetivo de avaliar o comportamento do parâmetro físico resistividade elétrica no solo após um vazamento de gasolina em subsuperfície, foi elaborado um experimento em laboratório, de modo a reproduzir um meio geológico natural.

O experimento consiste na adição de solo areno-argiloso pertencente à Formação Rio Claro, num tanque de vidro com $100 \mathrm{~cm}$ de comprimento, $40 \mathrm{~cm}$ de largura e $25 \mathrm{~cm}$ de altura (Figura 2). O solo foi analisado previamente por meio de peneiramento e análise granulométrica conjunta, que permitiu classificá-lo do como arenoso de granulação fina a média, com matriz siltosa.

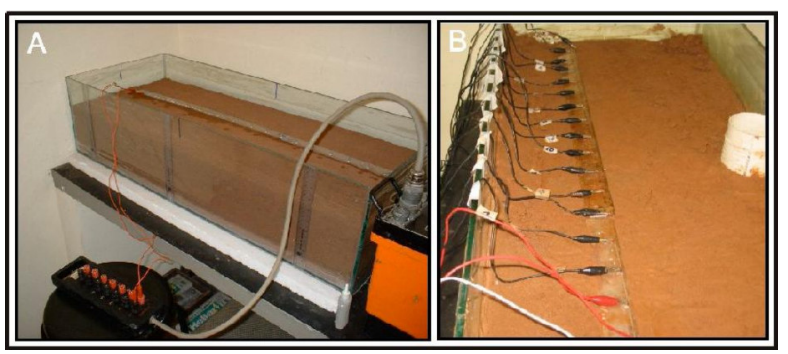

Figura 2 - Vista do tanque utilizado no experimento $(A)$ Arrarjo de eletrodos e poço de irijeção de gasolina (B)

Em trabalho de Braga \& Cardinali (2005), foram testados diversos tanques e espaçamentos para a aplicação da técnica de caminhamento elétrico em escala reduzida.

A proposta elaborada pelos autores supracitados é utilizada no presente trabalho, que consiste na aplicação

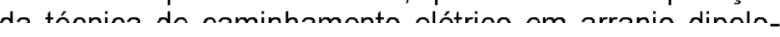
dinnlo nara 8 nívaic de invectirarãan em nrofundidade. Foram dispostos 17 eletrodos em linha e fixados numa régua a cada $5 \mathrm{~cm}$, num total de $90 \mathrm{~cm}$ de comprimento (Figura 2). Os eletrodos foram confeccionados em cobre e apresentam $2 \mathrm{~cm}$ de comprimento $0,2 \mathrm{~cm}$ de espessura.

A injeção de gasolina foi realizada por meio de um poço instalado no tanque, ranhurado em toda sua extensão e locado na lateral da linha de aquisição (Figura 2). Pncteriormente fni adicinnada ánı destilada no tannı até o estabelecimento de uma coluna d'água com $15 \mathrm{~cm}$ de altura, para simulação de nível freático, também foram adicionados dois litros de gasolina vendida em postos de combustíveis, que contem álcool etílico em sua composição. A injeção de gasolina foi realizada por meio de um poço instalado no tanque, ranhurado em toda sua extensão e locado na lateral da linha de aquisição (Figura 2).

Os valores de resistividade foram interpolados através do nrnnrama Surfer 8 nor mein do métndn de krinanem,

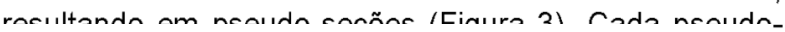
seção foi interpretada de maneira qualitativa, pois apresentam apenas a profundidade teórica de investigação.

\section{Análise e Interpretação dos Resultados}

Estabelecidos os requisitos previamente descritos, foi realizada uma linha de caminhamento elétrico para defininão dac nondirños natıraic dn material nII seia,

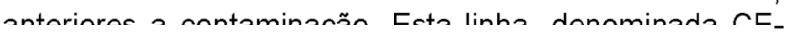
01, apresenta três horizontes principais paralelos e estratificados, que descrevem a zona não saturada (Alta resistividade, entre $90 \Omega \mathrm{m}$ e $200 \Omega \mathrm{m}$ ), franja capilar (resistividade intermediária, entre $40 \Omega \mathrm{m}$ e $90 \Omega \mathrm{m}$ ) e zona saturada (baixa resistividade, entre $3 \Omega \mathrm{m}$ e $40 \Omega \mathrm{m}$ ) (Figura 3). O nível freático é definido no contato entre a franja capilar e a zona saturada.

Após injeção de gasolina, foram realizadas leituras sucessivas de resistividade após 30m, 48h, 168h, 264h, 
408h, 672h, 792h, 1512h, 1824h e 2328h, também apresentados sob a forma de pseudo-seções (Figura 3).

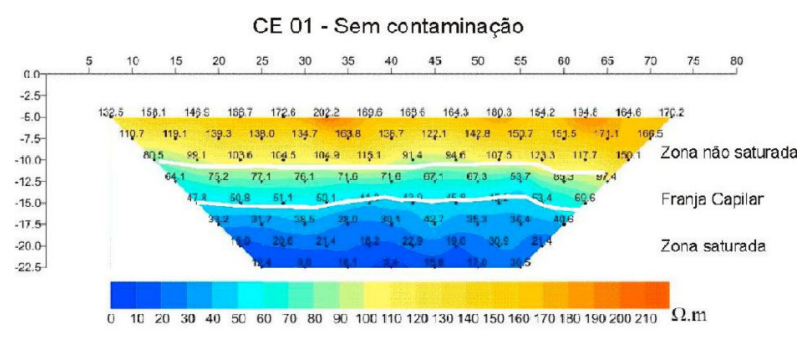

Figura 2 - Linha CE-01, com níveis de saturação do experimento.

A projeção lateral do poço de injeção de gasolina é realçada por traço em vermelho, na posição $45 \mathrm{~m}$ nas pseudo-seções realizadas após a contaminação

A pseudo-seção realizada 30 minutos após contaminação indica aumentos pontuais de resistividade na zona saturada e não saturada, com aparente perturbação dos horizontes anteriormente planos, com valores máximos de $350 \Omega m$ e $122 \Omega m$ respectivamente (Figura 3 ).

Após 48 horas é notável o aumento da resistividade em todos os horizontes, com extremos de $488 \Omega \mathrm{m}$ para a zona não saturada, $135 \Omega \mathrm{m}$ para a franja capilar e $150 \Omega \mathrm{m}$ para a zona saturada.

A seção realizada após 168 horas apresenta redução nos valores resistividade em todos os horizontes, com extremos de $285 \Omega \mathrm{m}$ para a zona não saturada, $166 \Omega \mathrm{m}$ para franja capilar e $66 \Omega \mathrm{m}$ para a zona saturada (Figura 3).

As seções que foram realizadas após 264 horas e 408 horas apresentam características semelhantes, com tendência de redefinicãn dos horizontes de satıracãn, valores médios bastantes elevados para a zona não saturada, e valores relativamente elevados para os demais horizontes

Esta tendência também é verificada nas seções realizadas após 672 horas, 792 horas, 1512 horas e 1824 horas. Nestes casos, ocorre uma pequena elevação nos valores médios de resistividade para a zona saturada.

Os valores obtidos após 2328 horas da contaminação indicam a tendência de retorno aos valores medidos no experimento sem contaminação, com resistividade relativamente mais elevada apenas para a zona não saturada.

Foi realizada uma análise estatística da resistividade aparente apresentada nos $2^{\circ}, 4^{\circ}, 6^{\circ}$ e $8^{\circ}$ níveis geoelétricos, para avaliação de variações ao longo do período de ensaios, por meio da média dos valores de cada nível geoelétrico em cada período de medição (Figura 4)

Outra característica apresentada em todos os níveis geoelétricos é a tendência de redução da resistividade após $1824 \mathrm{~h}$, pois os valores medidos $2328 \mathrm{~h}$ após a contaminação são próximos aos valores do ambiente não contaminado em quase todos os casos.

Para verificação desta tendência e estimativa do tempo necessário para retorno aos valores naturais de resistividade do ambiente contaminado, foram estabelecidas linhas de tendência e respectivas equações de reta apenas para a média dos valores de $1824 \mathrm{~h}$ e $2328 \mathrm{~h}$ (Figura 5)

nc recultadnc indicam serem nereccárias $24.30 \mathrm{~h} 2501 \mathrm{~h}$, $2604 \mathrm{~h}$ e $2883 \mathrm{~h}$ para retorno aos valores naturais de resistividade, respectivamente para o $2^{\circ}, 4^{\circ}, 6^{\circ}$ e $8^{\circ}$ níveis geoelétricos

\section{Discussão e Conclusões}

A variação no parâmetro resistividade elétrica apresentada ao longo período de experimento pode estar relacionada ao grau de degradação da gasolina.

Ocorreu aumento de resistividade imediatamente após contaminação, resultando em valores máximos após $264 \mathrm{~h}$ para a zona não saturada e após $48 \mathrm{~h}$ para a franja capilar e zona saturada. Tais diferenças podem estar relacionadas a processos distintos de percolação do contaminante para os horizontes de saturação.

A expulsão de ar contido nos poros da zona não saturada e seu preenchimento por gasolina aparentemente necessita de um tempo relativamente maior, quando comparado a percolação do produto na franja capilar e zona saturada, onde ocorrem processos de diluição e dispersão em meio aquoso.

Estes valores extremos são sucedidos por queda relativa na resistividade e estabilização. $O$ período de estabilização nos valores foi de $1560 \mathrm{~h}$ para a zona não saturada e de $1776 \mathrm{~h}$ para franja capilar e zona saturada.

A partir $1824 \mathrm{~h}$ até o ultimo ensaio a $2328 \mathrm{~h}$, ocorre queda nos valores. Como não houve novas injeções de gasolina é esperado que a ação de processos de degradação atuantes na gasolina resulte no retorno aos valores naturais de resistividade no meio.

As linhas de tendência obtidas a partir das duas últimas medidas, para o $2^{\circ}, 4^{\circ}, 6^{\circ}$ e $8^{\circ}$ níveis geoelétricos indicam períodos distintos para retorno aos valores naturais de resistividade (Figura 5).

Os resultados indicam uma relação crescente entre tempo de estabilidade e grau de saturação do solo. Os processos de atenuação ou degradação natural de compostos orgânicos são controlados principalmente pelo teor de oxigênio disponível para a ação de microorganismos.

O oxigênio consumido durante os processos de degradação nos horizontes secos ou de baixa umidade é renovado com relativa rapidez, devido a trocas gasosas com a atmosfera. Por outro lado, o oxigênio consumido a partir da água é reposto por absorção atmosférica de forma mais lenta, resultando na desaceleração nos processos de degradação. 
As variações de resistividade descrita ao longo do dispersão/diluição de gasolina no solo, respectivamente aos períodos de aumento e posterior queda e estabilidade dos valores de resistividade. Os processos de denradacãn nodem ter inícin a nartir de $1824 \mathrm{~h}$, quando começa haver queda nos valores de resistividade. O tempo necessário para retorno aos valores naturais de resistividade possivelmente estão associados ao teor de oxigênio disponível, que por sua vez estão condicionados ao grau de saturação do solo.

Os resultados indicam uma relação crescente entre tempo de estabilidade e grau de saturação do solo. Os processos de atenuação natural de compostos orgânicos são controlados principalmente pelo teor de oxigênio disponível para a ação de microorganismos.

O oxigênio consumido durante os processos de degradação nos horizontes secos ou de baixa umidade é renovado com relativa rapidez, devido a trocas gasosas com a atmosfera. Por outro lado, o oxigênio consumido a partir da água é reposto por absorção atmosférica de forma mais lenta, resultando na desaceleração nos processos de degradação

As variações de resistividade descrita ao longo do dispersão/diluição de gasolina no solo, respectivamente aos períodos de aumento e posterior queda e estabilidade dos valores de resistividade. Os processos de denradarãn nndem ter inírin a nartir de 1824h, quando começa haver queda nos valores de resistividade. O tempo necessário para retorno aos valores naturais de resistividade possivelmente estão associados ao teor de oxigênio disponível, que por sua vez estão condicionados ao grau de saturação do solo.

\section{Agradecimentos}

Os autores agradecem também ao técnico Francisco Manuel Garcia Barrera, do Departamento de Geologia Aplicada, UNESP - Rio Claro, pelo auxílio nas aquisições dos dados.

\section{Referências Bibliográficas}

Atekwana F A Saıık W A Werkema.Ir $\cap \cap 20 n n$. Investigations of geoelectrical signature at a hydrocarbon contaminated site. Journal of Applied Geophysics, $n^{0} 44$ : $167-180$

Atekwana F A Werkema .Ir $\cap \cap$ Allen .IP Smart, L.A., Duris, J.W., Atekwana, E.A., Cassidy, D.P., Sauck,
W.A. \& Rossback, S., 2004. Evidence for Microbial

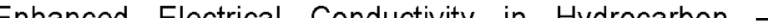
Contaminated Sediments. Geophysical Research Letters. Vol. 31.

Cassidv חP Werkema .Ir $\cap \cap$ Saıırk W A., Atekwana, E.A., Rossback, S.; Duris, J., 2001. The Effects of LNAPL Biodegradation Products on Electrical Conductivity Measurements. Journal of Environmental and Engineering Geophysics. Vol. 6: 47-52.

EPA - United States Environmental Protection Agency. , 1998. Technical Protocol for evaluating natural attenuation of chlorinated solvents in ground water Washington DC, epa/600: 98-128.

FPA - I Inited States Fnvirnnmental Protertion Arenry., 2004. Use of monitored natural attenuation at superfund, RCRA corrective action, and Underground Storage Tank Sites. Office of Solid Waste and Emergency Response, Directive 9200, 9: 4 - 17 .

Lima, O. A. L., Sato, H. K, Porsani, M. J., 1995. Imaging indıstrial contaminant nlımes with resistivitv terchniaııes. Journal Applied Geophysics, n 34: 93-108.

Rabus, R. \& Heider, J., 1998. Initial reactions of anaerobic metabolism of alkylbenzenes in denitrifying and

170: $377-394$

Sauck, W. A., 2000. A model for the resistivity structure of LNAPL plumes and their environs in sandy sediments. Applied Geophysics, $n^{0} 44$ : 151-165.

Vega, M., Osella, A. A., Lascano, E., 2003. Joint inversion

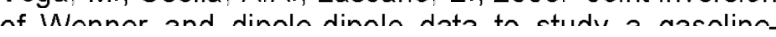
contaminated soil. Journal of Applied Geophysics, Amsterdam, vol. 54: 97-109

Werkema Jr., D.D., Atekwana E.A., Endres, A. L., Sauck, W.A., Cassidy, D.P., 2003. Investigating the geoelectrical response of hydrocarbon contamination undergoing hindenradation Gennhvsical Research l etters Vol 3n, $n^{0} 12$.

Wilson, S. C. \& Jones, K. C., 1993. Bioremediation of soils contaminated with Polynuclear Aromatic Hvdrncarhnne (PAHc) a review/ Fnvirnnmental Pollution, vol. 81: 229-249. 


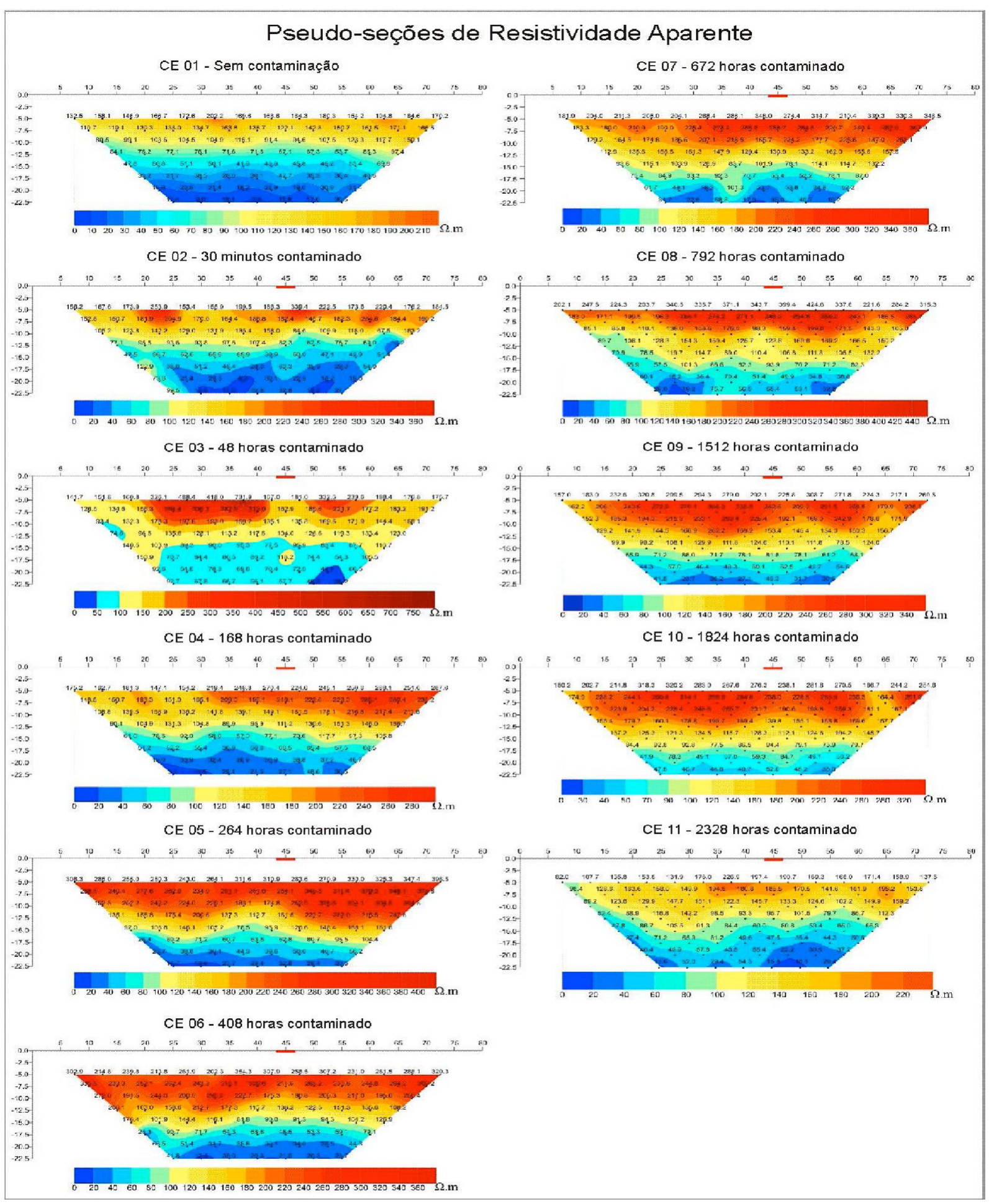

Figura 3 - Pseudo-seções de resistividade aparente 


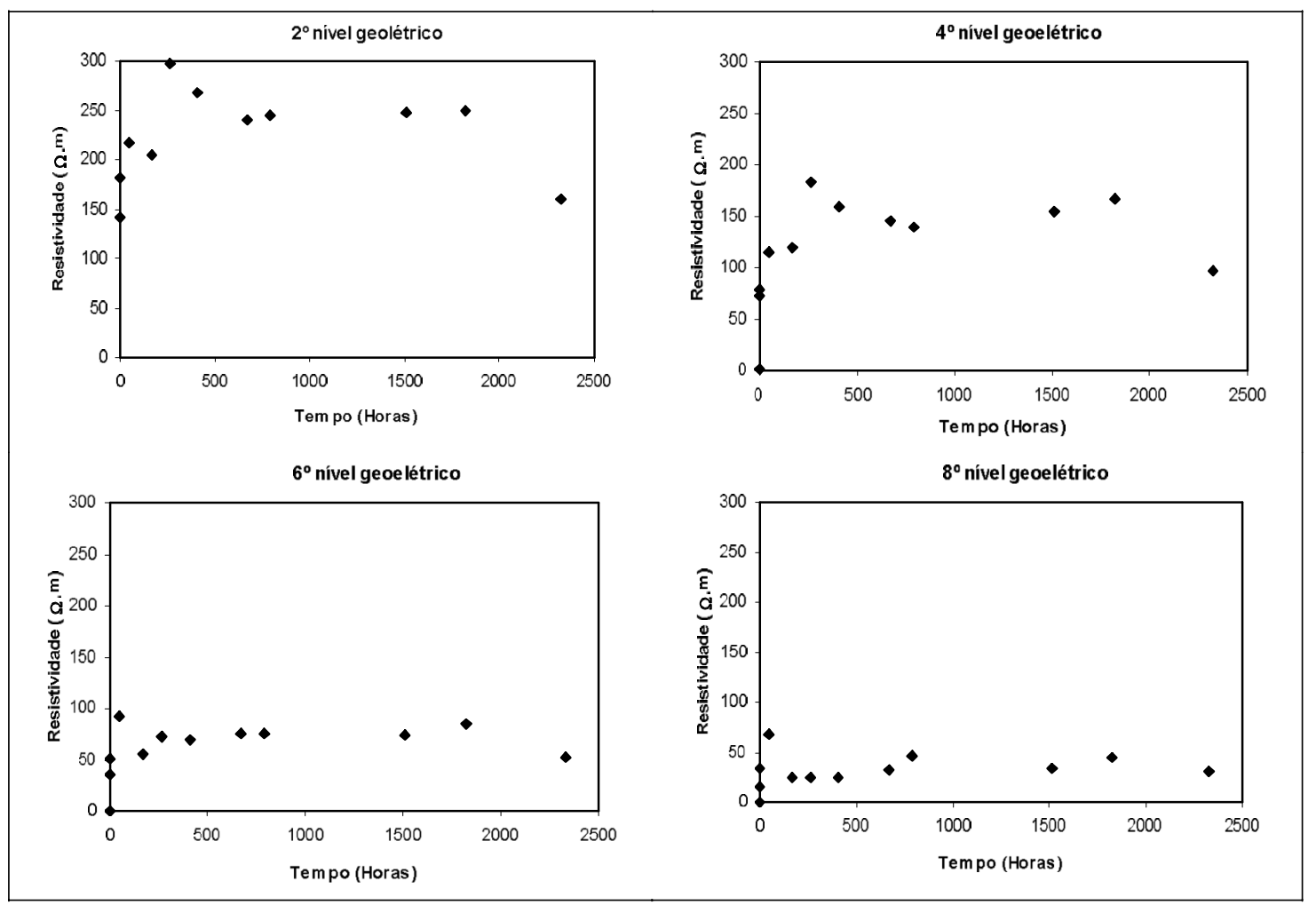

Figura 4 - Média da resistividade por nível geoelétrico, obtidos durante os diversos intervalos de tempo medidos

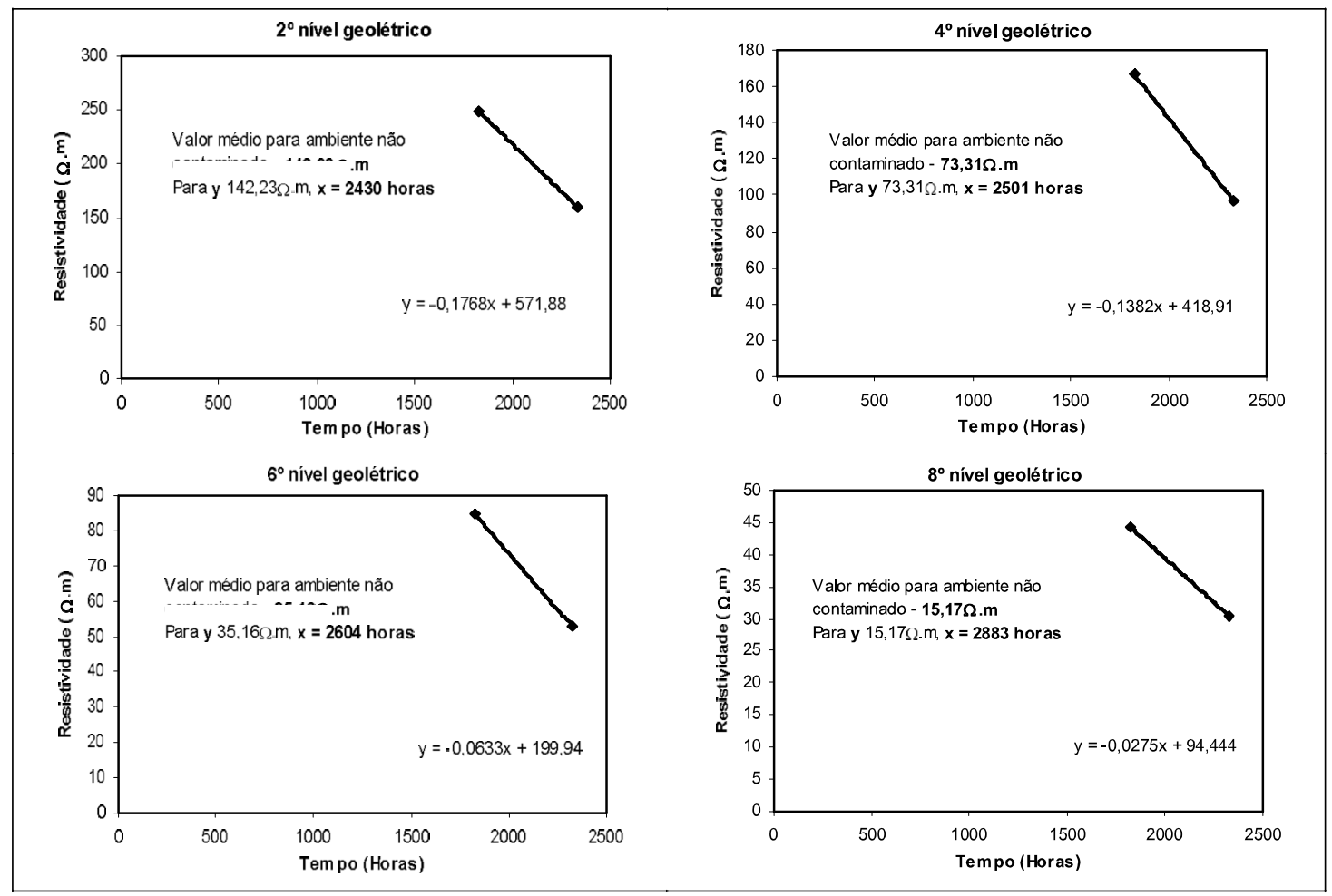

Figura 5 - Linhas de tendência, equações de reta e tempo previsto para retorno da resistividade aos valores naturais 